\title{
Synthesis and Molecular Dynamics Simulation of Amphiphilic Low Molecular Weight Polymer Viscosity Reducer for Heavy Oil Cold Recovery
}

\author{
Chao Ma ${ }^{1, *(1)}$, Xingyu Liu ${ }^{1}$, Longlong Xie ${ }^{1}$, Yan Chen ${ }^{1}$, Wendong Ren ${ }^{1}$, Wen Gu ${ }^{1}$, Minghua Zhang ${ }^{2}$ \\ and Huili Zhou ${ }^{3}$ \\ 1 School of Petroleum Engineering, Yangtze University, Wuhan 430100, China; \\ 202072354@yangtzeu.edu.cn (X.L.); 201972109@yangtzeu.edu.cn (L.X.); 202072349@yangtzeu.edu.cn (Y.C.); \\ 202072404@yangtzeu.edu.cn (W.R.); 202072358@yangtzeu.edu.cn (W.G.) \\ 2 State Key Laboratory of Polymer Materials Engineering, Sichuan University, Chengdu 610065, China; \\ zhangminghua851250@scu.edu.cn \\ 3 Wuhan Changde Energy Technology Co., Ltd., Wuhan 430100, China; huilizhou1980@126.com \\ * Correspondence: 500526@yangtzeu.edu.cn; Tel.: +86-138-7239-3959
}

Citation: Ma, C.; Liu, X.; Xie, L.; Chen, Y.; Ren, W.; Gu, W.; Zhang, M.; Zhou, H. Synthesis and Molecular Dynamics Simulation of Amphiphilic Low Molecular Weight Polymer Viscosity Reducer for Heavy Oil Cold Recovery. Energies 2021, 14, 6856. https://doi.org/10.3390/en14216856

Academic Editor: Kun Sang Lee

Received: 8 August 2021

Accepted: 15 October 2021

Published: 20 October 2021

Publisher's Note: MDPI stays neutral with regard to jurisdictional claims in published maps and institutional affiliations.

Copyright: (c) 2021 by the authors. Licensee MDPI, Basel, Switzerland. This article is an open access article distributed under the terms and conditions of the Creative Commons Attribution (CC BY) license (https:// creativecommons.org/licenses/by/ $4.0 /)$.

\begin{abstract}
In order to reduce the viscosity of heavy oil, the performance of emulsifying viscosity reducers is limited. In this study, a new kind of amphiphilic low molecular weight viscosity reducer was prepared by emulsion copolymerization of acrylamide (AM), acrylic acid (AA), 2-acrylamido2-methylpropanesulfonic acid (AMPS), and Butene benzene (PB). The synthesis feasibility and viscosity reduction mechanism of viscosity reducer in heavy oil were explored using Materials Studio software from the perspective of molecular dynamics. The results of the molecular dynamics simulation revealed that the addition of viscosity reducer into heavy oil varied the potential energy, non-potential energy, density and hydrogen bond distribution of heavy oil. Benefiting from its structure, the benzene ring in PB was well embedded in the interlayer structure of asphaltene, contributing to weaken the network structure of the heavy oil. Moreover, the two strong polar groups $\left(\mathrm{COO}^{-}\right.$and $\left.\mathrm{SO}_{3}{ }^{-}\right)$of AA and AMPS, which constituted the branched chains of the viscosity reducer's molecular structure, gradually disassembled the network structure from the 'inward' to the 'outward' of the heavy oil network structure, thereby driving heavy oil viscosity reduction (as clarified by molecular dynamics). Owing to its temperature resistance, this kind of new amphiphilic low molecular copolymer could be an effective viscosity reducer for heavy oil cold recovery at elevated temperatures.
\end{abstract}

Keywords: molecular dynamics simulation; amphiphilic polymers; hydrophobic monomer; viscosity reduction of heavy oil; viscosity reduction mechanism

\section{Introduction}

China is one of the world's major heavy oil producing countries with abundant geological reserves. More than 70 heavy oil fields have been discovered in the Junggar Basin, Songliao Basin, Nanxiang Basin, and Erlian Basin, and another 12 large and medium-sized oil basins and depressions have been discovered with proven oil-in-place of about 4 billion tons [1]. With depleting conventional light oil reserves, unconventional resources are gaining relevancy for balancing the growing demands of energy. Heavy oil resources, as one of the most relevant unconventional resources, are trapped within underground rocks [2]. Nevertheless, the heavy oil is rich of asphaltene, resin, and paraffin. They aggregate together into macromolecules, which results in high viscosity and poor fluidity as a consequence of compromising the flow and production process [3-5]. The heavy oil viscosity typically ranges from 50 to $50,000 \mathrm{mPa}$.s at reservoir temperature and pressure [6-11]. Therefore, reducing the heavy oil viscosity is a prerequisite for improved 
fluidity and transportation. As the commonly used methods, including steam huff and puff and steam assisted gravity drainage (SAGD), used for reduction of heavy oil viscosity are of low efficiency, coupled with the use of various equipment and challenging temperature corrosion, the entire process of heavy oil recovery has always been costly [12].

Heavy oil cold recovery is also regarded as a relevant heavy oil recovery technology. The key mechanism behind this method is based on the chemical interactions driving the viscosity reduction of heavy oil as a result of achieving rheological control. This ultimately improves the heavy oil recovery efficiency. Thanks to the simple process, less equipment required, and facile production management, heavy oil cold recovery technology has been used in some heavy oil blocks in the United States, Canada, and so on [13,14]. The chemical agents used for heavy oil cold recovery enable favorable thinning efficiency, stable dispersion, and good oil cleaning capability since they contain surface-active agents. It has been reported that while interacting with crude oil, the extent of aggregation of asphaltene is lowered by solvent dilution as to promote a considerable dispersion of asphaltene through depolymerizer. Subsequently, the formation of micronized crude oil occurs in the presence of surfactant to induce efficient viscosity reduction of crude oil $[15,16]$. Due to its various merits, including cost-efficiency, effortless operation, rapid effect, and good thinning effect, emulsifying viscosity reduction technology has been extensively used since the 1960s [17-19]. The emulsifying capability of surfactant is a critical factor affecting the viscosity reduction effect. It has been reported that, at a temperature higher than the cloud point of non-ionic surfactant, phase separation of surfactant occurs due to its reduced solubility in heavy oil, which results in a gradual loss of the viscosity reduction effect $[20,21]$. In a case of anionic surfactants, they mostly phase separate from crude oil, and the molecular structure is identical to that of the inherent surfactant in heavy oil. Under this circumstance, the formation of stable water-in-oil emulsion to reduce the heavy oil viscosity is much easier. However, inherent drawback of poor salinity tolerance, especially for petroleum sulfonate induces its precipitation when interacting with high valence cations in the formation as a consequence to mitigate the viscosity reducing effect of surfactant $[22,23]$.

The key mechanism of emulsifying viscosity reduction relies on the fact that the surfactant can turn the oil emulsion from $\mathrm{W} / \mathrm{O}$ to $\mathrm{O} / \mathrm{W}$ structure which sufficiently reduces the viscosity of heavy oil $[24,25]$. However, the contribution of surfactant on switching the emulsification from $\mathrm{W} / \mathrm{O}$ into $\mathrm{O} / \mathrm{W}$ structure is often compromised due to strong dependence of the surfactant from the 'outward' to 'inward' on dense-planar network structure of heavy oil [26]. With this mind, if a hydrophobic group containing benzene structure can be introduced into the 'internal' of a heavy oil network structure, the interactions between asphaltene molecules would be lowered, and the dense-planar asphaltene structure and resin would be segregated. Simultaneously, the presence of strong polar groups would imbue the heavy oil with various anisotropies as a result of effectively driving an efficient viscosity reduction effect. In this work, from the perspective of the characteristics of heavy oil network structure, a low molecular weight viscosity reducer was synthesized by emulsion copolymerization using AM as a central-chain, and PB, AMPS, and AA as a branched-chains of the copolymer as shown in Figure 1. According to the principle of solubility, the benzene ring in PB would be embedded in the interlayer structure of asphaltene as a result to weaken the network structure of heavy oil. The two strong polar monomers (AA and AMPS) which constituted the branched-chains of copolymeric materials would be introduced into the heavy oil. Consequently, the strong hydrophilicity of the sulfonic acid group in AMPS would not only be beneficial to effectively maintain the O/W structure, but also to disassemble the network structure from the 'inward' to 'outward' of the heavy oil network structure as illustrated in Figure 2, driving an effective viscosity reduction effect. It was worth noting that this molecular structure was inspired from the author's previous study who found that the amphiphilic copolymer whose molecular structure was constituted of AM, and n-butylstyrene could favorably adsorbed the emulsified oil droplets in heavy oil wastewater [27-29]. In addition, the Materials Studio 5.0 molecular simulation 
software was used to quantitatively monitor the feasibility of synthesis of viscosity reducer from the perspective of molecular dynamics and to simulate the interaction between heavy oil and viscosity reducer, and to explore the viscosity reduction mechanism in heavy oil. Based on molecular dynamics, an amphiphilic low molecular weight copolymer was synthesized as a viscosity reducer in heavy oil.

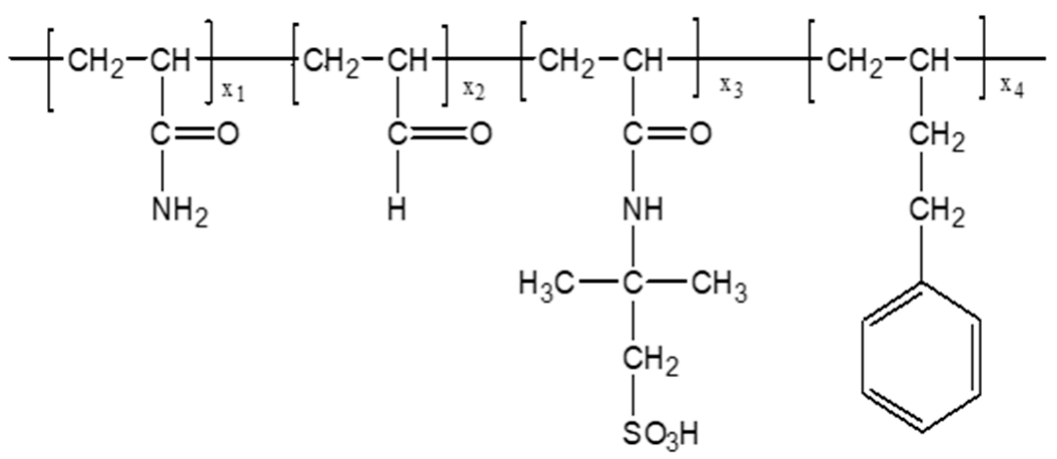

Figure 1. Structure diagram of polymer.

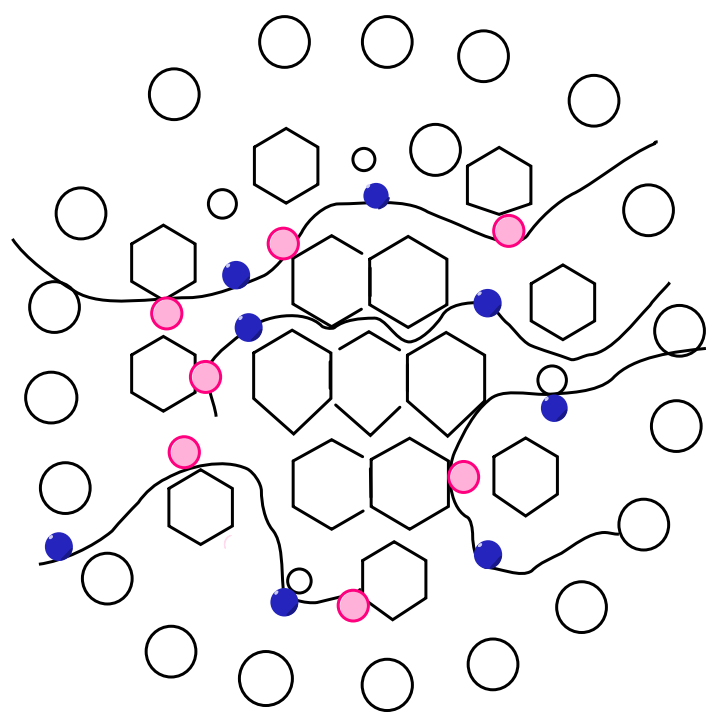

Figure 2. Network structure of heavy oil and mechanism of viscosity reduction of amphiphilic polymer. $\bigcirc$ Hydrophobic group containing benzene ring; $\bullet$ Carboxyl group/amide group/sulfonic acid group; O Water molecule; $\bigcirc$ Heavy oil.

\section{Materials and Methods}

\subsection{Materials and Instruments}

Acrylamide (AM, 99.9\%), acrylic acid (AA, 99.5\%), 2-acrylamido-2-methylpropanesulfonic acid (AMPS, 99\%) were obtained from Tianjin Beilian Fine Chemical Development Co., Ltd, Tianjin, China. Butene benzene (PB, 98.9\%) was from Beijing Bailingwei Technology Co., Ltd, Beijing, China. Ammonium persulfate $\left[\left(\mathrm{NH}_{4}\right)_{2} \mathrm{SO}_{4}, 99.5 \%\right]$, sodium sulfite $\left(\mathrm{Na}_{2} \mathrm{SO}_{3}\right.$, $99.5 \%$ ), sodium hydroxide $(\mathrm{NaOH}, 96 \%)$, tween- 80 , petroleum ether (boiling point $30-69^{\circ} \mathrm{C}$ ), dichoromethane $\left(\mathrm{CH}_{2} \mathrm{Cl}_{2}, 99.5 \%\right)$, chloroform $\left(\mathrm{CHCl}_{3}, 99.5 \%\right)$, acetone $(99.5 \%)$, anhydrous ethanol (99.7\%), mercaptan (99.5\%), and isopropanol (99.5\%) were procured from Tianjin Fuchen chemical reagent factory, Tianjin, China. Kerosene, neutral alumina (100-200 mesh, specific surface area $>150 \mathrm{~m}^{2} / \mathrm{g}$, pore volume: $0.23 \mathrm{~cm}^{2} / \mathrm{g}-0.27 \mathrm{~cm}^{2} / \mathrm{g}$ ), glass indicator adsorption column, muffle furnace were purchased from Wuhan Zhongtian Chemical Co., Ltd, Wuhan, China. The degassed heavy oil sample, denoted as SZ36-1 was obtained from Suizhong Oilfield, offshore, China. Fourier transform infrared spectrometer: colet 560, TA, New Castle, USA; Thermo gravimetric analyzer (SDTQ600, DuPont, New Castle, USA); Room 
temperature gel chromatograph, PL-GPC50, Agilent, CA, USA; Brookfield DV3T viscometer, Bolfe, MA, USA.

\subsection{Molecular Dynamics Simulation Test}

\subsubsection{Establishment of Heavy Oil Structure Model}

Firstly, the composition of SZ36-1 was examined according to SH/T0509-2010 “Determination of petroleum asphaltene components" and SY/T 6282-1997" [30] and "Analytical approach of fluid physical property for heavy-oil reservoirs-measurements of porous flow rheological characterization for crude oil" [24,25]. In detail, the analytical pure reagents were re-distilled in a round-bottomed flack equipped with a fractionating column, refluxing condenser and collector, while heated around the boiling point. Subsequently, the neutral alumina was activated in a porcelain evaporating dishes and heated at $500{ }^{\circ} \mathrm{C}$ for $6 \mathrm{~h}$. Afterwards, $50 \mathrm{mg}$ of dehydrated heavy oil was dissolved with $30 \mathrm{~mL}$ of re-distilled petroleum ether, then a precipitate of asphaltene was removed by filtration, and dried. The asphaltene was adsorbed with activated alumina and placed into glass adsorption column. The saturated hydrocarbons, aromatic hydrocarbons and colloid species were washed with 40 60 mL re-distilled petroleum ether, mixture of dichloromethane and petroleum ether $(v / v, 1 / 2)$, and mixture of chloroform and anhydrous ethanol $(v / v, 1 / 1)$, respectively. The solvents were removed using rotary vacuum dryer, and the products were dried in a vacuum oven under constant temperature.

Secondly, Materials Studio 5.0 software was used to obtain a total of 16 representative molecular structures of 4 distinctive heavy oil samples in the 3D Atomistic document [31], and the molecular number proportion to scale modeling was adjusted in Amorphous cell. The temperature, number of cycles, and initial density were fixed at $298 \mathrm{~K}, 10$, and $0.6 \mathrm{~g} / \mathrm{cm}^{3}$, respectively. The structural modeling of heavy oil was optimized from three distinctive aspects including, energy, geometry, and dynamic as illustrated in Figure 3.

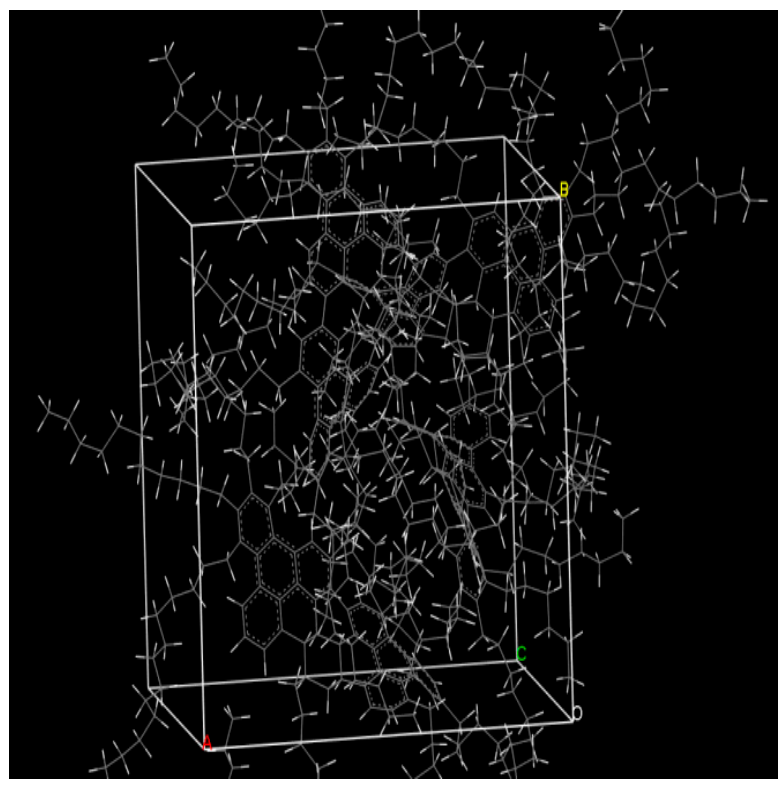

Figure 3. Conformation of multi-component optimization model for heavy oil.

\subsubsection{Kinetic Simulation of Viscosity Reducer Synthesis Process}

In this study, the molecular models of AM, AA, AMPS, and PB were established in 3D Atomistic document. The structure and performance of the obtained copolymers were optimized from three distinctive aspects including, energies, geometries, and dynamics in the Forcite calculation module to ensure the consistency in molecular structure and performance of copolymer with the desired one as depicted in Figure 4. After designing and optimizing the copolymers molecular structure in the polymer builder tools, the mole ratio of AM:AA:AMPS:PB = 30:2:3:3, chain length of 10, and chain segment of 1 were then 
obtained. Subsequently, the Amorphous cell module (temperature of $333 \mathrm{~K}$ and number of cycles of 10) was employed to establish the viscosity reducer model and the calculation was carried out using Forcite analysis. The dynamic simulation of viscosity reduction mechanism of copolymer in heavy oil was therefore established. The simulation was performed in the Compass force field using the ratio of heavy oil to viscosity reducer of $1: 1$, the temperature was controlled at $333 \mathrm{~K}$, with cycle number of 10 , and the optimized spatial structure of the interaction between viscosity reducer and heavy oil was obtained as represented in Figure 5.

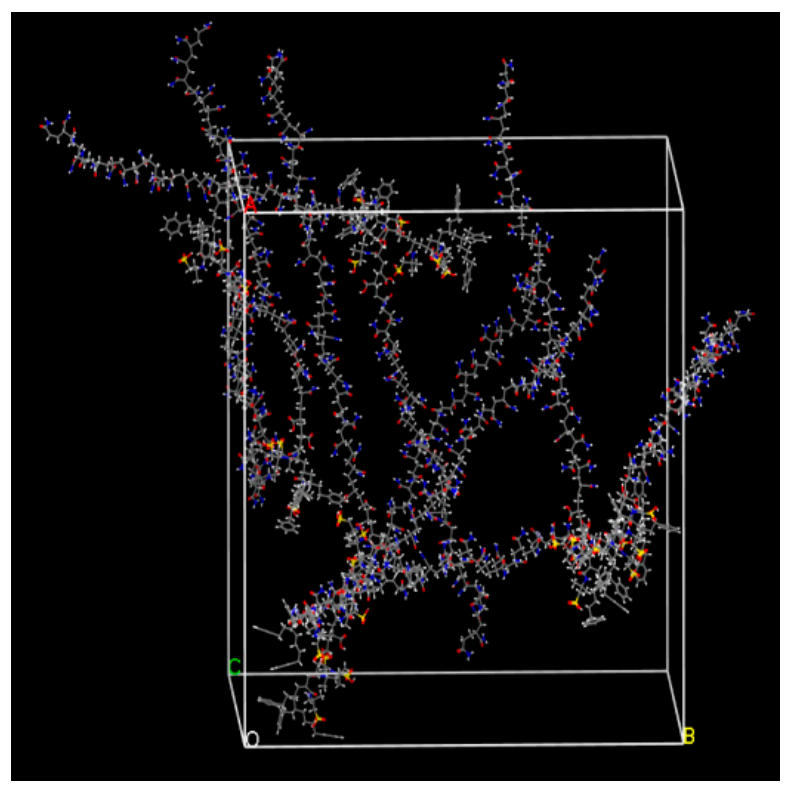

Figure 4. Establishment of monomer and viscosity reducer structure.

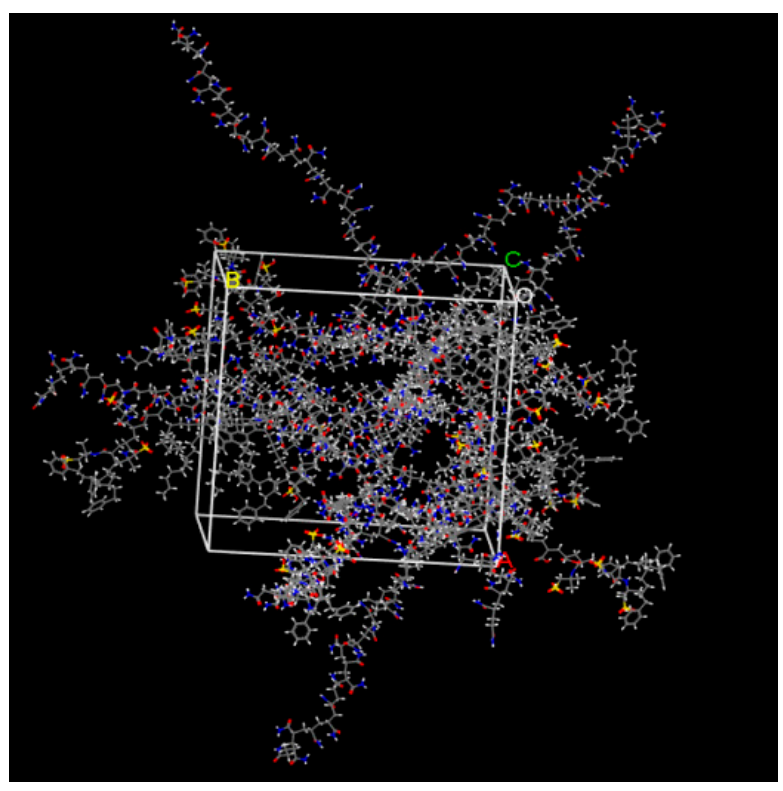

Figure 5. Heavy oil and its structure after interaction with viscosity reducer.

\subsubsection{Synthesis of Viscosity Reducer Synthesis Method of Viscosity Reducer}

Prior to synthesis of viscosity reducer by emulsion polymerization, a stable emulsion (mass ratio of kerosene:deionized water $=\mathrm{T}-80: 1: 4: 0.006$ ) was first prepared. Then, the requisite amounts of AM:AA:AMPS:PB were introduced into the three-necked flask according to the mole ratio of 50-60:4-6:3-9:6-9, and stirred for about 30 min to ensure 
complete dispersion of monomers with the $\mathrm{pH}$ of the mixture maintained between 9-10. Once the components were uniformly dispersed, $0.01-0.05 \%$ (based on total monomers mass) of chain transfer agents (mercaptan and isopropyl alcohol) were added, followed by nitrogen bubbling for $30 \mathrm{~min}$. Subsequently, $1-1.5 \%$ of KPS (based on total monomers mass) was added to the mixture and the reaction was conducted at $60^{\circ} \mathrm{C}$ under continuous nitrogen atmosphere purging for $8 \mathrm{~h}$. Once the reaction terminated, the product was washed 3-5 times with a mixture of acetone/ethanol $(2 / 1, v / v)$, dried at $80^{\circ} \mathrm{C}$, and crushed into powder. The optimal mole ratio of AM:AA:AMPS:PB $=$ 60:5:6:9 was used to prepare the viscosity reducer $\left(\mathrm{P}_{2}\right)$.

\subsubsection{Characterization and Viscosity Reduction Effect}

FT-IR spectra was taken on Nicolet 560 FT-IR analyzer in the frequency range of $4000-400 \mathrm{~cm}^{-1}$. The thermogravimetry analysis (TGA) was performed Dupont SDTQ600 thermogravimetry analyzer with heating rate of $20 \mathrm{~K} / \mathrm{min}$ from room temperature to $600{ }^{\circ} \mathrm{C}$ under $\mathrm{N}_{2}$ atmosphere. The molecular weight was measured on Agilent gel chromatography analysis system at $30^{\circ} \mathrm{C}$, the aqueous solution $0.1 \mathrm{~mol} / \mathrm{L}$ was used as the mobile phase at a flow rate of $1 \mathrm{~mL} / \mathrm{min}$ and the injection volume was $50 \mu \mathrm{L}$.

A certain amount of $\mathrm{P}_{2}$ was added into the heavy oil, stirred for $20 \mathrm{~min}$ at room temperature, and the apparent viscosity of mixture was examined by Brookfield DV3T rheometer (Brookfield Engineering Lab, Bolfe, MA, USA) at a desired temperature.

\section{Results}

\subsection{Establishment of Heavy Oil Structure Model}

Heavy oil is composed primarily of asphaltenes, and colloidal macromolecule compounds, but the specific composition is still unclear. At present, two mainstream approaches are used to establish the microscopic model of heavy oil. The first one refers to the construction of model based on the average molecular structure [31]. In this way, the B-L method is employed to draw the average molecular structure of the four components in heavy oil regarding the data from hydrogen spectrum and X-ray diffraction, and the complex molecular structure can be transformed into four distinctive structures. However, this does not picture the specific molecular structure of heavy oil. Hence, the complex interaction in-between heavy oil molecules cannot be simulated. The second one lies on the construction of physical properties model which is identical to that of heavy oil by exploiting the known four representative molecules [32]. After analyzing the composition of heavy oil, four main components including, saturated hydrocarbons, aromatic hydrocarbons, resins and asphaltene constituted the heavy oil as listed in Table 1. Referring to [33], a total of 16 molecular structures representing the 4 components of heavy oil were obtained. Based on the actual composition of heavy oil, the number of molecules of each component was adjusted so that the composition of the constructed heavy oil model was consistent with that of SZ36-1 as shown in Table 2. The heavy oil model was therefore established using the method described in Section 2.2.1.

Table 1. Composition of SZ36-1.

\begin{tabular}{ccc}
\hline Component & Unit & Content \\
\hline Saturated hydrocarbon & $\%$ & 52.15 \\
content & $\%$ & 27.10 \\
Aromatics content & $\%$ & 31.70 \\
Resin & $\%$ & 7.68 \\
Asphalt & $\mathrm{mPa}$ & 1134.5 \\
Viscosity of heavy oil $\left(50^{\circ} \mathrm{C}\right)$ & ${ }^{\circ} \mathrm{C}$ & 44.5 \\
Pour point of crude oil & & \\
\hline
\end{tabular}


Table 2. Molecular composition of heavy oil model.

\begin{tabular}{ccc}
\hline Component & Representative Component Number & Number of Molecules \\
\hline \multirow{3}{*}{ Asphaltene } & 1 & 2 \\
& 2 & 2 \\
& 3 & 3 \\
\hline \multirow{2}{*}{ Colloid } & 4 & 4 \\
& 5 & 8 \\
& 6 & 8 \\
& 7 & 4 \\
Aromatics & 8 & 2 \\
hydrocarbon & 9 & 2 \\
& 10 & 3 \\
\hline \multirow{2}{*}{ Saturated hydrocarbon } & 11 & 6 \\
& 12 & 7 \\
& 13 & 9 \\
\hline
\end{tabular}

Data from the reference [34].

\subsection{Dynamic Simulation of Viscosity Reducer Synthesis Process}

As earlier described in Section 2.2.2, both temperature variation and total energy variation were simulated during the synthesis process of viscosity reducer, and a total of 50 dynamic models were acquired as indicated in Figures 6 and 7. From the curve of temperature variation represented in Figure 6, it can be observed that the temperature fluctuated in small range close to the set synthesis temperature $(333 \mathrm{~K})$, which revealed that the set temperature of the periodic system had little influence on the simulated results. The curve of variation of the total energy was also presented in Figure 7. It was found that, at a fixed reaction temperature of $333 \mathrm{~K}$, the total energy of the system also slightly fluctuated within a small range. We could not only observe that this fluctuation occurred around $670 \mathrm{kcal} / \mathrm{mol}$, but also the energy of C-C double bond was $145.7 \mathrm{kcal} / \mathrm{mol}$, inferring that under this reaction condition, the rupture of $C-C$ double bond could be envisioned, and the polymerization reaction was therefore theoretically feasible.

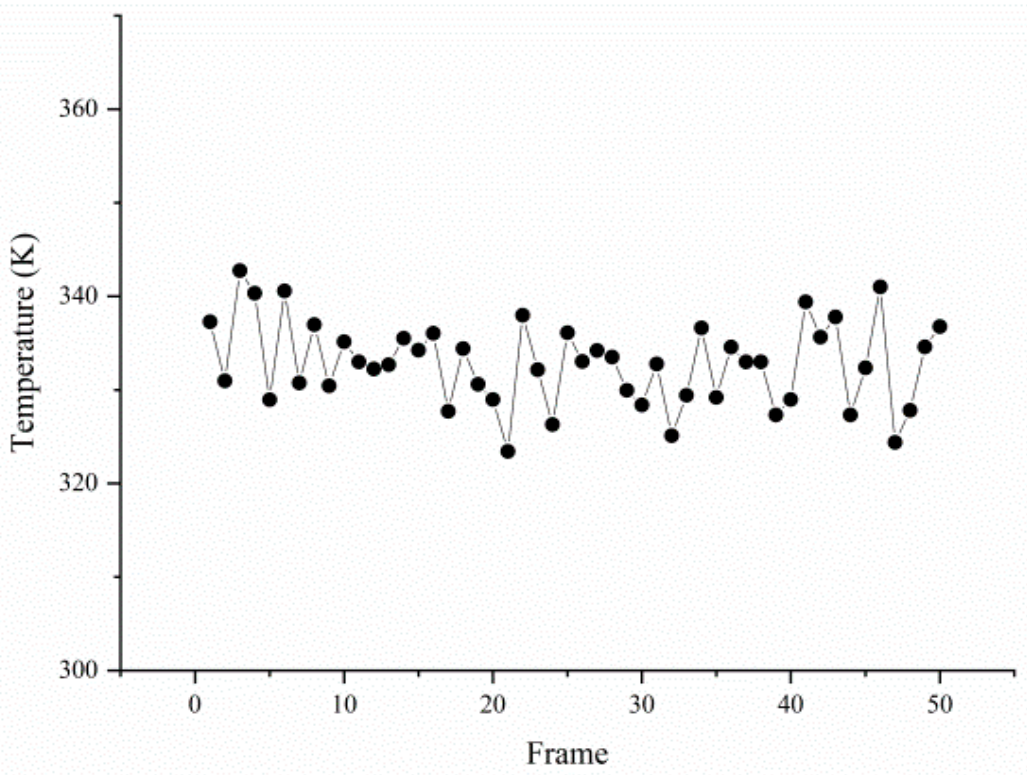

Figure 6. Temperature variation of the system during the synthesis of viscosity reducer. 


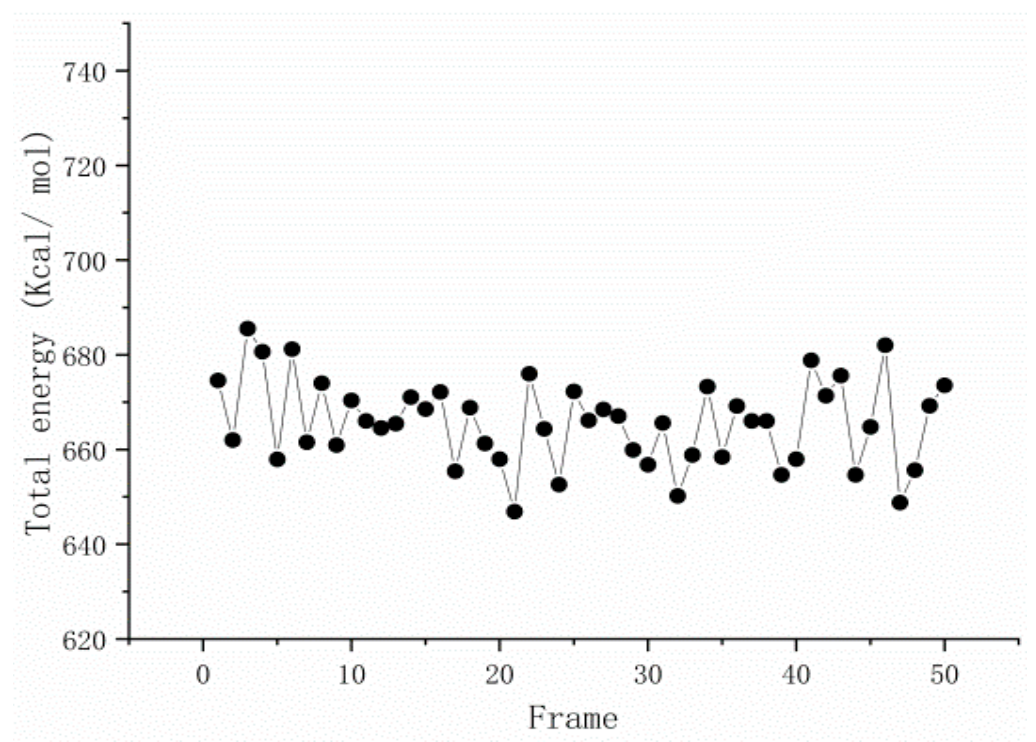

Figure 7. Temperature variation of system energy during the synthesis of viscosity reducer.

\subsection{Dynamic Simulation of Viscosity Reduction Mechanism}

As it can be observed in Figure 8, compared to the corresponding curve of heavy oil, that of the viscosity reducer with heavy oil significantly increased. This sharp variation in potential energy could be due to the attachment of asphaltene and resin molecules to the viscosity reducer which resulted in the forming of a large spatial steric hindrance which was constituted of an abundant number of branched-chain structures of the viscosity reducer molecules. It can be inferred that the viscosity reducer molecules which was constituted of a sulfonic group, carboxyl group, and hydrophobic structure with benzene ring mutually induced strong adsorption of benzene ring structure in asphaltene and colloid molecules. From the same Figure, we could also observe an obvious increase in negativity of nonpotential energy which might be attributed to the two strong polar monomers (AA and AMPS) as a consequence of intensifying the interaction between the viscosity reducer and inward of heavy oil. As a matter of fact, the strong hydrophilicity of the sulfonic group in AMPS was beneficial to maintain the $\mathrm{O} / \mathrm{W}$ emulsion via the formation of more stable hydrogen bonding. Subsequently, the network structure was gradually disassembled from the "inward" to "outward" of the heavy oil structure. Under such circumstances, the long carbon chain in AMPS, asphaltene, and colloid molecules were longitudinally extended resulting in the formation of planar and easily combed linear structure, thus preventing the occurrence of three-dimensional assembly structure and eventually the viscosity reduction. From Figure 9, it was noticed that the overall density of both heavy oil and viscosity reducer with heavy oil fluctuated within a small range. However, compared to the overall density of heavy oil $\left(1.00 \mathrm{~g} / \mathrm{cm}^{3}\right)$, that of the viscosity reducer with heavy oil $\left(0.96 \mathrm{~g} / \mathrm{cm}^{3}\right)$ was slightly lower, translating that the asphaltene and colloid compounds in heavy oil were removed. Thus, this phenomenon contributed not only to lowering the density of heavy oil, but also the declining of heavy oil viscosity could be envisioned (which will be investigated later). 


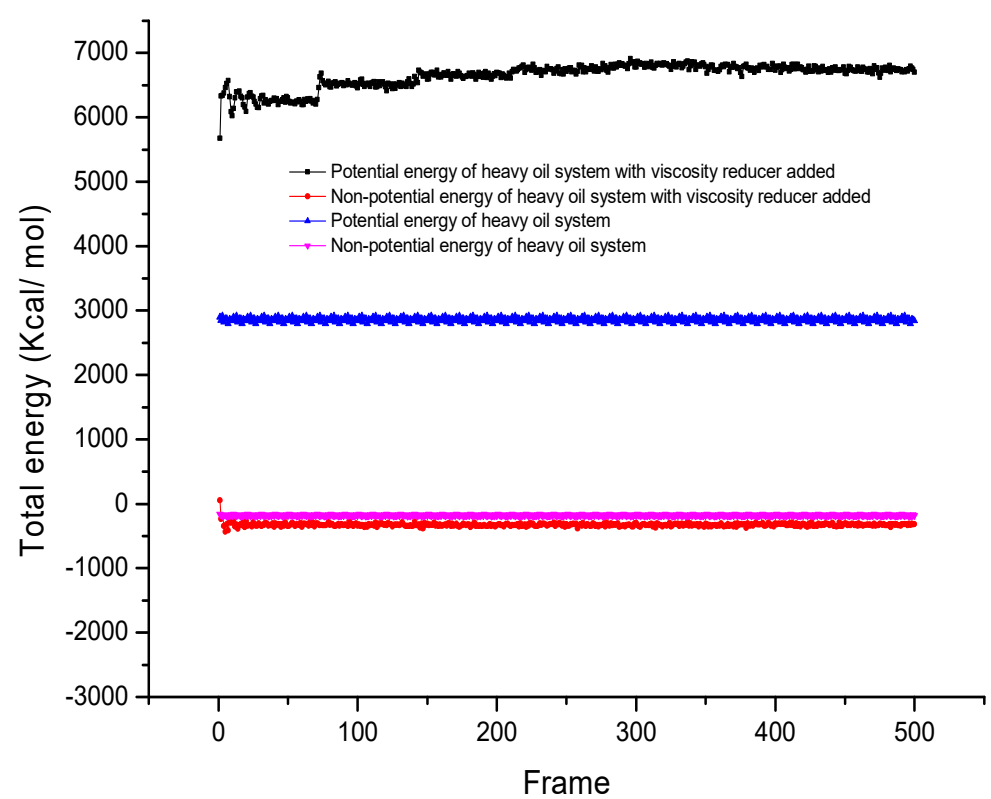

Figure 8. Influence of viscosity reducer on potential energy and non-potential energy of heavy oil.

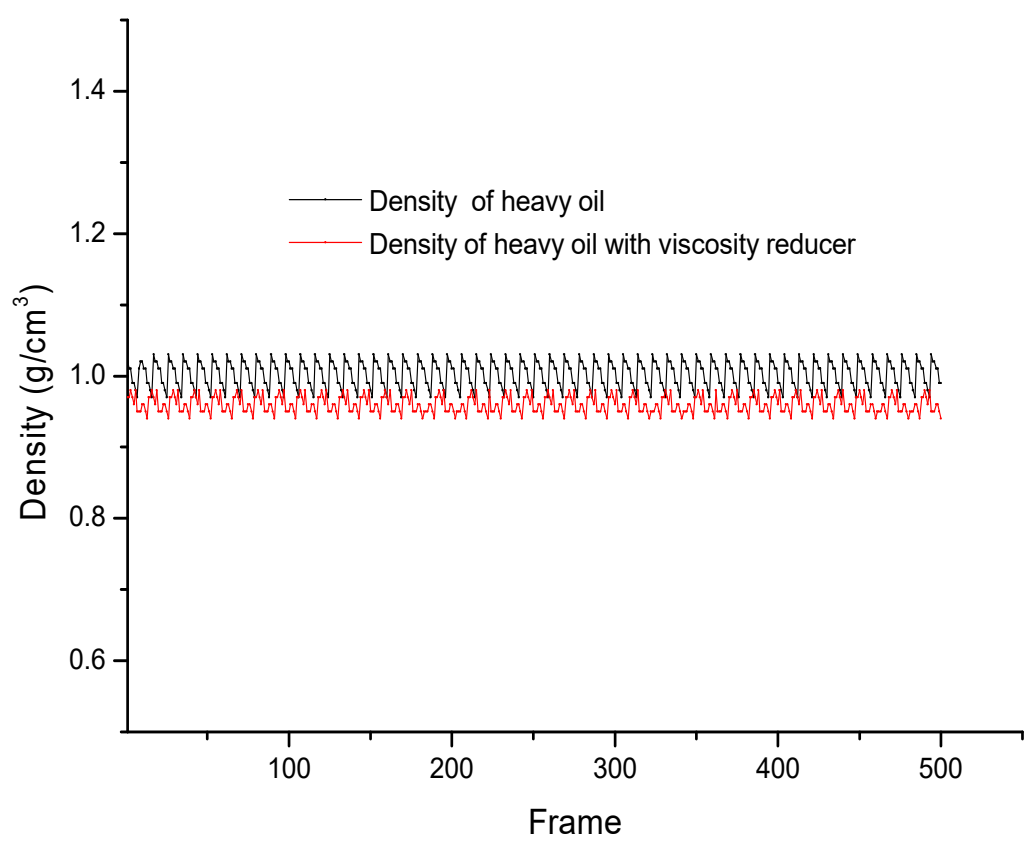

Figure 9. Influence of viscosity reducer on density of heavy oil.

It is generally believed that asphaltenes molecules are shaped planar and have dense aromatic rings, side-chains, and a large number of heteroatoms which facilitate the susceptibility to undergo hydrogen bonding and self-aggregation. Moreover, the basic structure of resin molecules is based on the aromatic system composed of multiple rings and there are surrounded by naphthenic rings. Both aromatic rings and naphthenic rings are imbued with several normal or isoalkyl side chains of distinctive lengths, and their molecular weight, polarity, and susceptibility to undergo hydrogen bonding are all second to asphaltene [35]. The length of hydrogen bond distribution of heavy oil and in the presence of viscosity reducer were investigated and indicated in Figure 10. As it can be observed, the hydrogen bond of heavy oil was mainly distributed between 1.11-1.14 E (A), which accounted for about $43 \%$, followed by $1.53-1.55 \mathrm{E}$ (A), which represented about $25.7 \%$. These findings suggested that there was not only a large number, but also a stable hy- 
drogen bonds in the heavy oil. From the corresponding curve of the length of hydrogen bond distribution of heavy oil with viscosity reducer shown in the same figure, we could determine that the hydrogen bonds were mainly distributed between 1.1-1.11 E (A), which accounted for almost $30 \%$, followed by $1.53-1.57 \mathrm{E}$ (A) with a rate of $6.9 \%$ compared to the length of hydrogen bond distribution of heavy oil, which in the presence of viscosity reducer exhibited multiple distribution peaks. This could be ascribed to the amphiphilic function of the viscosity reducer, which contributed to break down the "planar" structure in heavy oil as a result to weaken the interaction between asphaltene and resin. It is worth noting that, compared to the hydrogen bond distribution in the heavy oil, in the presence of viscosity reducer was slightly wider, mainly attributed to a significant decrease in the proportion, indicating that the viscosity reducer can significantly reduce the distribution proportion of hydrogen bonds in the heavy oil. After collapsing the rigid structure, in addition to the viscosity reducer, various components were involved in the formation of hydrogen bonding, contributing to effectively reduce the viscosity of heavy oil.

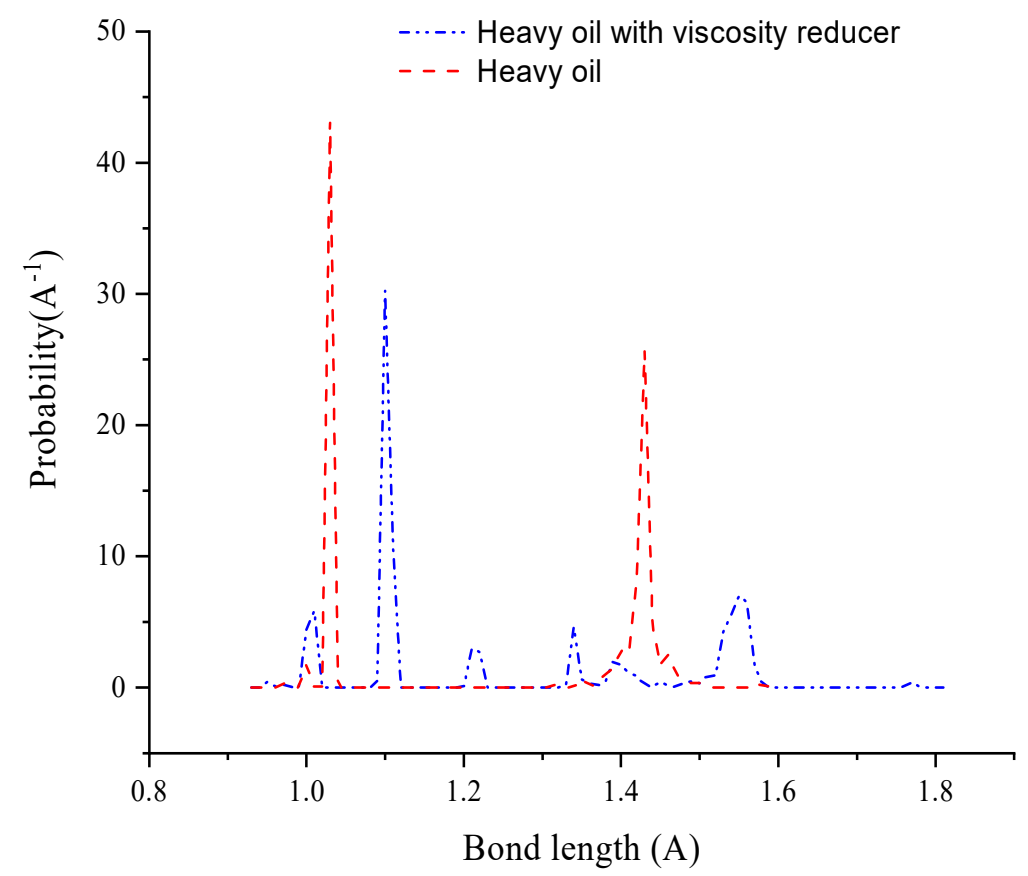

Figure 10. Distribution of the length of hydrogen bond in heavy oil and heavy oil with viscosity reducer.

\subsection{Structure and Performance Characterization of Viscosity Reducer}

\subsubsection{IR Characterization of Viscosity Reducer}

Figure 11 shows the FT-IR spectra of viscosity reducer. The large band at $3417.77 \mathrm{~cm}^{-1}$ corresponded to the stretching vibration of $\mathrm{N}-\mathrm{H}$ in amide. The stretching vibration of $\mathrm{C}=\mathrm{O}$ in amide was assigned to the peak at $1673.39 \mathrm{~cm}^{-1}$. The peak observed in the range of $1337.71-1045.62 \mathrm{~cm}^{-1}$ was attributed to the stretching vibration of C-N in amide. The $\mathrm{COO}^{-}$in the acrylic acid vibrated at $1554.38 \mathrm{~cm}^{-1}$. The three adsorption peaks recorded at $1194.10 \mathrm{~cm}^{-1}, 1118.10 \mathrm{~cm}^{-1}$, and $1042.6 \mathrm{~cm}^{-1}$ were ascribed to the $\mathrm{SO}_{3}{ }^{-}$ groups in AMPS [36]. The broad absorption peak noticed at $622.54 \mathrm{~cm}^{-1}$ was due to the absorption characteristic of benzene ring. In the light of these results, the four monomers were involved in the copolymerization reaction. 


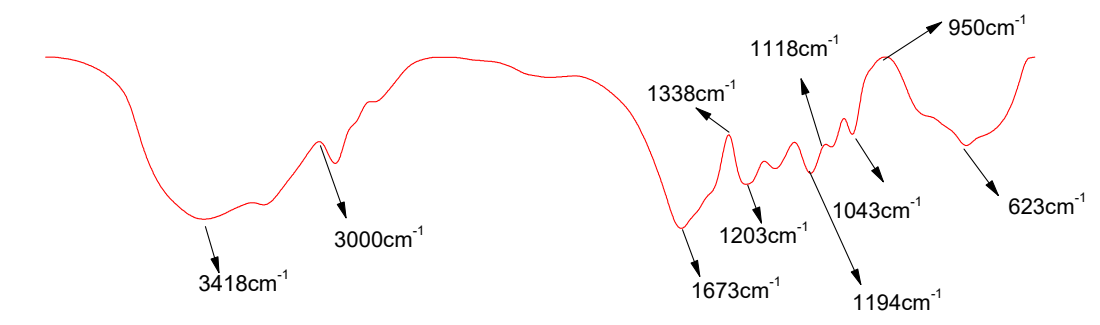

\begin{tabular}{|c|c|c|c|c|c|c|c|}
\hline${ }_{4000}^{\prime}$ & $\begin{array}{c}1 \\
3500\end{array}$ & 3000 & $\begin{array}{c}1 \\
2500\end{array}$ & 2000 & $\begin{array}{c}1 \\
1500\end{array}$ & 1000 & $\frac{1}{500}$ \\
\hline
\end{tabular}

Figure 11. FT-IR spectrum of viscosity reducer.

\subsubsection{TGA Analysis of Viscosity Reducer}

The TGA analysis curve of viscosity reducer and polyacrylamide taken from room temperature to $600{ }^{\circ} \mathrm{C}$ in $\mathrm{N}_{2}$ atmosphere was displayed in Figure 12. The first region below $100{ }^{\circ} \mathrm{C}$ with a large slope was ascribed to elimination of water molecules and unreacted monomers. The initial decomposition temperature was recorded as $250{ }^{\circ} \mathrm{C}$, while that of polyacrylamide was $210{ }^{\circ} \mathrm{C}$ [36], suggesting that the viscosity reducer had superior temperature resistance. This was mainly due to introduction of benzene ring structure of PB and sulfonic group which contributed to improve the thermal stability of viscosity reducer, thus indicating that the viscosity reducer could find use in high temperature heavy oil formation.

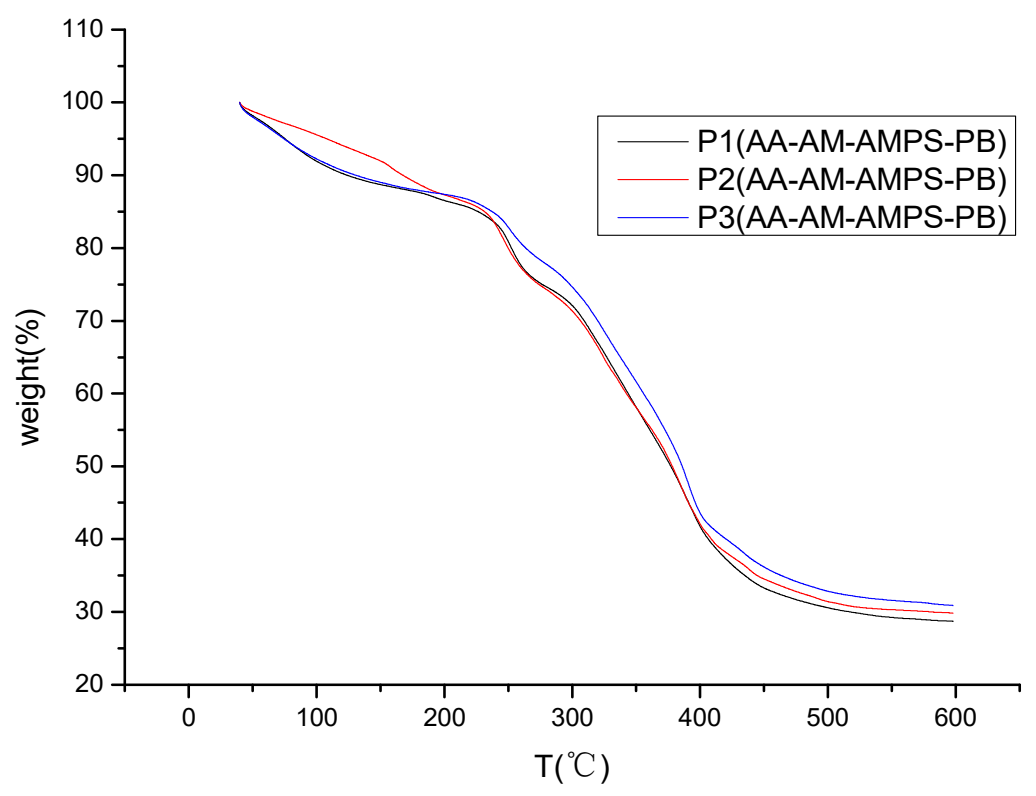

Figure 12. TGA curve of viscosity reducer.

\subsubsection{Molecular Weight Test of Viscosity Reducer}

As determined by gel chromatography method, the results of molecular weight of viscosity reducer were listed in Table 3. It can be observed that, the weight-average molecular weight $(\mathrm{Mw})$ of viscosity reducer varied between $8-100,000$. The low molecular weight enables the dispersion of viscosity reducer in heavy oil, which favor the facile migration into the 'inward' of heavy oil as a result to better weaken, and eventually destroy, the network structure of the heavy oil. 
Table 3. Results of molecular weight of viscosity reducer samples.

\begin{tabular}{|c|c|c|c|}
\hline Sample & Symbol & Meaning & Value \\
\hline \multirow{3}{*}{$\begin{array}{c}\text { P1 } \\
(\mathrm{AA}-\mathrm{AM}-\mathrm{AMPS}-\mathrm{PB})\end{array}$} & Mw & The weight average molecular weight & 83,567 \\
\hline & $\mathrm{Mn}$ & The number average molecular weight & 59,423 \\
\hline & $\mathrm{Mw} / \mathrm{Mn}$ & $\begin{array}{l}\text { The ratio of weight average molecular weight } \\
\text { and number average molecular weight }\end{array}$ & 1.420 \\
\hline \multirow{3}{*}{$\begin{array}{c}\mathrm{P} 2 \\
(\mathrm{AA}-\mathrm{AM}-\mathrm{AMPS}-\mathrm{PB})\end{array}$} & Mw & The weight average molecular weight & 94,563 \\
\hline & $\mathrm{Mn}$ & The number average molecular weight & 48,765 \\
\hline & $\mathrm{Mw} / \mathrm{Mn}$ & $\begin{array}{l}\text { The ratio of weight average molecular weight } \\
\text { and number average molecular weight }\end{array}$ & 1.930 \\
\hline \multirow{3}{*}{$\begin{array}{c}\text { P3 } \\
(\mathrm{AA}-\mathrm{AM}-\mathrm{AMPS}-\mathrm{PB})\end{array}$} & Mw & The weight average molecular weight & 84,789 \\
\hline & $\mathrm{Mn}$ & The number average molecular weight & 56,328 \\
\hline & $\mathrm{Mw} / \mathrm{Mn}$ & $\begin{array}{l}\text { The ratio of weight average molecular weight } \\
\text { and number average molecular weight }\end{array}$ & 1.510 \\
\hline
\end{tabular}

Note: $\mathrm{P}_{1}(\mathrm{AA}: \mathrm{AM}: \mathrm{AMPS}: \mathrm{PB})$ = 50:4:3:6; $\mathrm{P}_{2}$ (AA:AM:AMPS:PB) = 60:5:6:9; $\mathrm{P}_{3}$ (AA:AM:AMPS:PB) = 55:5:6:7.

\subsubsection{Effect of Viscosity Reducer on Heavy Oil Viscosity}

In order to ensure the fluidity of heavy oil, the experimental temperature was maintained at $70{ }^{\circ} \mathrm{C}$, distinctive concentration of $\mathrm{P}_{2}$ solutions was added into the heavy oil and the final viscosity was taken. Figure 13 revealed the contribution of viscosity reducer concentration on the viscosity of heavy oil. As it can be observed, with increasing $\mathrm{P}_{2}$ concentration from 0 to $0.5 \mathrm{wt} . \%$, the viscosity of heavy oil dropped by almost $86.14 \%$, as to reflect its favorable viscosity reduction effect. As a matter of fact, the viscosity reducer $\left(\mathrm{P}_{2}\right)$ had a low molecular weight and sufficiently long molecular chain. Moreover, the molecular structure of $\mathrm{P} 2$ was composed of strong polar groups $\mathrm{COO}^{-}(\mathrm{AM}, \mathrm{AA})$ and $\mathrm{SO}_{3}{ }^{-}$ (AMPS), which aided the migration of $\mathrm{P}_{2}$ into the 'inward' of heavy oil as a consequence to disassemble, but also to effectively oppose the water molecules from entering the internal structure. Thus, the $\mathrm{O} / \mathrm{W}$ structure predominated over the $\mathrm{W} / \mathrm{O}$ one as a consequence to favor the fluidity of heavy oil. In this way, the benzene ring structure which constituted the $\mathrm{P}_{2}$ molecular structure was well embedded in the asphaltene interlayer [37]. From the same plot, we could notice that increasing the $\mathrm{P}_{2}$ concentration contributed to gradually increase the apparent viscosity of heavy oil, which might be due to entanglement of the long chain of viscosity reducer in the $\mathrm{O} / \mathrm{W}$ emulsion.

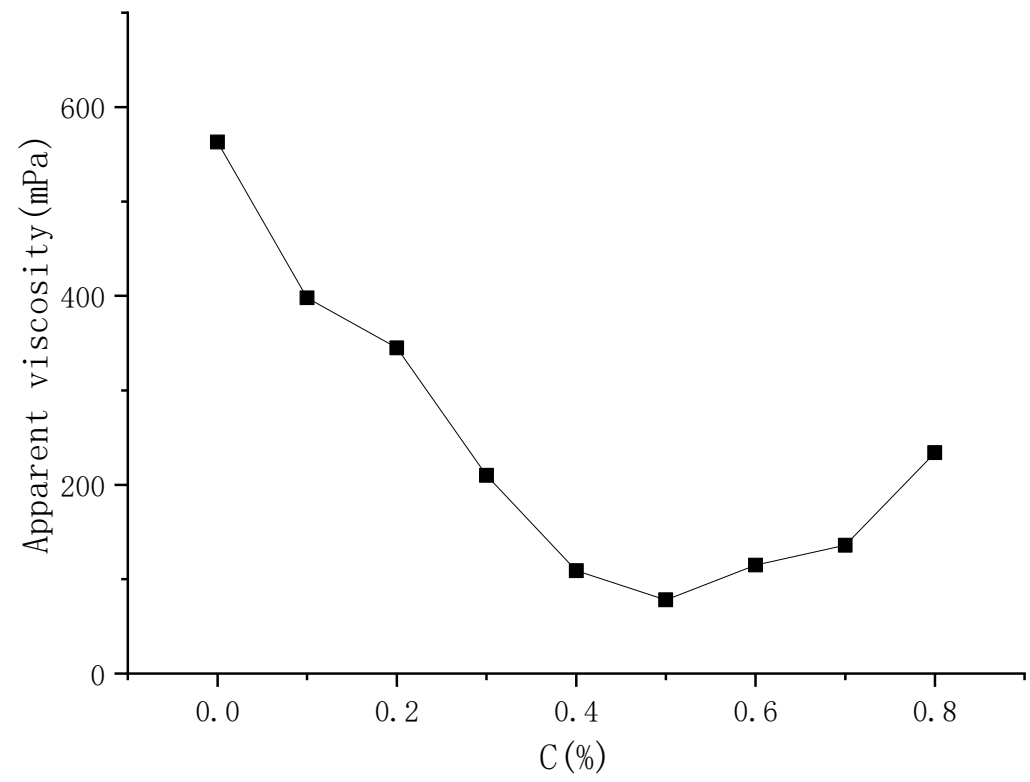

Figure 13. Effect of viscosity reducer on apparent viscosity of heavy oil. 


\section{Conclusions}

In this study, considering SZ36-1 as the research object, a combination of experimental method and molecular dynamic simulation method were employed to establish the structural model of heavy oil, and dynamic simulation of viscosity reduction mechanism. The feasibility of the synthesis method of low molecular weight amphiphilic polymer viscosity reducer was confirmed by molecular dynamics and the molecular structure was characterized. By introducing the hydrophobic benzene ring and strong hydrophilic groups into the polymer molecular chain, the amphiphilic low viscosity reducer could interact with the 'inward' resulting to imbue the 'outward' with various anisotropies, which weakened and destroyed the dense network structure of heavy oil and eventually drive the viscosity reduction of heavy oil. Thus, with increasing low molecular weight concentrations from 0 to $0.5 \%$, the heavy oil viscosity reduced by about $86.14 \%$ at $70{ }^{\circ} \mathrm{C}$. The experimental results also proved that low molecular weight amphiphilic structure viscosity reducer exhibited good viscosity reduction effect. For heavy oil of SZ36-1 (formation temperature at $\left.50-65^{\circ} \mathrm{C}\right)$, the polymer can meet the need for heavy Oil Cold Recovery, which has a good reference for the flowable heavy oil.

Author Contributions: Conceptualization, L.X. and W.R.; data curation, Y.C. and W.G.; methodology C.M. and M.Z.; investigation, H.Z. and X.L. All authors have read and agreed to the published version of the manuscript.

Funding: This research was funded by Xinjiang Autonomous Region Natural Science Fund ProjectStudy on Synthesis and Oil Displacement Mechanism of Porous Hyperbranched Heavy Oil ColdCollecting and Viscosity-Release Oil Displacement Agent (Grant No.: 2019D01A91) and the Opening Project of State Key Laboratory of Polymer Materials Engineering (Sichuan University) (Grant No. sklpme2018-4-36).

Conflicts of Interest: The authors declare no conflict of interest.

\section{References}

1. Xu, G.R.; Li, X.; Xie, K. Mechanism and oil dis-placement of heavy oil emulsification and viscosity reducer: A case study on heavy oil reservoir and fluid conditions of Bohai oilfield. J. Petrochem. Univ. 2016, 29, 57-62.

2. Xiang, Y.C.; Ning, W.; Shu, Q.X. Research progress and development trend of heavy oil emulsifying viscosity reducer: A review. Pet. Sci. Technol. 2021, 39, 550-563. [CrossRef]

3. Martínez-Palou, R.; Mosqueira, M.D.L.; Zapata-Rendón, B.; Mar-Juárez, E.; Bernal-Huicochea, C.; Clavel-López, J.D.L.C.; Aburto, J. Transportation of heavy and extra-heavy crude oil by pipeline: A review. J. Pet. Sci. Eng. 2011, 75, 274-282. [CrossRef]

4. Alimohammadi, S.; Zendehboudi, S.; James, L. A comprehensive review of asphaltene deposition in petroleum reservoirs: Theory, challenges, and tips. Fuel 2019, 252, 753-791. [CrossRef]

5. Fakher, S.; Ahdaya, M.; Elturki, M.; Imqam, A. Critical review of asphaltene properties and factors impacting its stability in crude oil. J. Pet. Explor. Prod. Technol. 2020, 10, 1183-1200. [CrossRef]

6. Sun, J.H.; Zhang, F.S.; Wu, Y.W.; Liu, G.L.; Li, X.N.; Su, H.M.; Zhu, Z.Y. Overview of emulsified viscosity reducer for enhancing heavy oil recovery. IOP Conf. Ser. Mater. Sci. Eng. 2019, 479, 012009. [CrossRef]

7. Mai, A.; Bryan, J.; Goodarzi, N.; Kantzas, A. Insights Into Non-Thermal Recovery of Heavy Oil. J. Can. Pet. Technol. 2009, 48, 27-35. [CrossRef]

8. Babadagli, T.; Er, V.; Naderi, K.; Burkus, Z.; Ozum, B. Use of Biodiesel as an Additive in Thermal Recovery of Heavy Oil and Bitumen. J. Can. Pet. Technol. 2010, 49, 43-48. [CrossRef]

9. Alvarado, V.; Manrique, E. Enhanced Oil Recovery: An Update Review. Energies 2010, 3, 1529-1575. [CrossRef]

10. Su, B.; Fujimitsu, Y. Research on Heavy Oil Thermal Recovery by $\mathrm{CO}_{2}$ Steam Flooding with Help of Combination of BoreholeSurface Electric Potential and Cross-Borehole Electric Potential. Energy Explor. Exploit. 2011, 29, 797-815. [CrossRef]

11. Babadagli, T.; Ozum, B. BioDiesel as Additive in High Pressure and Temperature Steam Recovery of Heavy Oil and Bitumen. Oil Gas Sci. Technol.-Rev. d'IFP Energ. Nouv. 2012, 67, 413-421. [CrossRef]

12. Zhang, Y.B.; Wang, N.; Xia, N.; Huan, S.X.; Yan, D. Evaluation and mechanism analysis of emulsion viscosity reduction performance of enhanced dispersion system. J. Petrochem. Univ. 2016, 29, 18-22.

13. Zhang, L.; Pan, Z.; Zhang, Z.; Yan, S.L.; Wen, J.; Chen, S. Thermodynamic and Economic Analysis Between Organic Rankine Cycle and Kalina Cycle for Waste Heat Recovery From Steam-Assisted Gravity Drainage Process in Oilfield. J. Energy Resour. Technol. 2018, 140, 122005. [CrossRef]

14. Maity, S.K.; Ancheyta, J.; Marroquín, G. Catalytic Aquathermolysis Used for Viscosity Reduction of Heavy Crude Oils: A Review. Energy Fuels 2010, 24, 2809-2816. [CrossRef] 
15. Roscioli, J.R.; Herndon, S.C.; Yacovitch, T.I.; Knighton, W.B.; Zavala-Araiza, D.; Johnson, M.R.; Tyner, D.R. Characterization of methane emissions from five cold heavy oil production with sands (CHOPS) facilities. J. Air Waste Manag. Assoc. 2018, 68, 671-684. [CrossRef] [PubMed]

16. Wang, T.T.; Lu, X.G.; Cao, W.J.; Zhang, Y.B.; Wang, N.; Xia, H. Viscosity reduction effect and mechanism of heavy oil surfactant emulsification-Bohai BZ25-1s reservoir as an example. J. Pet. Chem. Univ. 2017, 30, 26-31.

17. Kang, W.L.; Liu, Y.L.; Meng, L.W. Screening of spontaneous emulsified viscosity reducer for heavy oil in Yongping Oilfield and evaluation of oil displacement effect. Pet. Geol. Recovery Effic. 2012, 1, 59-61.

18. Zhang, X. Synthesis and Solution Properties of Microblock Amphiphilic Polymers; Southwest Petroleum University: Chengdu, China, 2015.

19. Jia, H.; Huang, P.; Han, Y.; Wang, Q.; Wei, X.; Huang, W.; Dai, J.; Song, J.; Yan, H.; Liu, D. Synergistic effects of Janus graphene oxide and surfactants on the heavy oil/water interfacial tension and their application to enhance heavy oil recovery. J. Mol. Liq. 2020, 314, 113791. [CrossRef]

20. Chen, L.; Zhang, Q.; Jiang, Y. Discussion on the status quo and application of heavy oil non-heating gathering technology. Nat. Gas Pet. 2010, 1, 6-9.

21. Duan, H.B.; Liu, Q.; Luo, W.Y. Study on Viscosity Reduction Technology and Mechanism of Tahe Heavy Oil. Chem. Eng. 2013, 12, 26-30.

22. Fan, J.L.; Wu, B.F.; Li, Y. Study on Emulsification and Viscosity Reduction of Henan Super Heavy Oil. Oilfield Chem. 2014, 3, 451-456.

23. Li, M.R.; Qi, L.Y.; Wang, W.; Lin, T.S.K. Study on Emulsification and Viscosity Reduction Mechanism of Shengli Super Heavy Oil. J. Fuel Chem. 2013, 6, 679-684.

24. Yu, L.L. Preparation and Performance Evaluation of Polymer Heavy Oil Viscosity Reducer; Jilin University: Jilin, China, 2012.

25. Zhang, L.J.; Yue, X.G.; Guo, Z.J. Study on Emulsification Characteristics of ASP System and Dagang and Daqing Crude Oils. Pet. Geol. Recovery Effic. 2010, 3, 74-76.

26. Cui, G.S. Study on Emulsification and Viscosity Reduction Method and Mechanism of Heavy Oil; China University of Petroleum: Beijing, China, 2009.

27. Ma, C.; Zhu, Q.; Pan, M.L. Discussion on flocculation of temperature sensitive polymer in heavy oil wastewater. Ind. Water Treat. 2017, 37, 50-53.

28. Ma, C.; Pan, M.L.; Su, C. The oil displacement effect of a temperature sensitive polymer solution containing benzene ring structure. Appl. Chem. Ind. 2017, 46, 1047-1050.

29. Ma, C.; Wang, G.; Zhang, X. Synthesis and Solution Properties of Temperature-Sensitive Copolymer OBS/DEAM/AM. Polym. Mater. Sci. Eng. 2016, 32, 28-32.

30. Test method for separation of asphalt into four fractions. Oil and Gas Industry Standard Assay for Determination of Petroleum Asphaltene Components. (SH /T 0509-2010).

31. Wu, C.; Zhang, R.S.; Zhang, Z.G.; Sui, Z.Y.; Wu, Z.B. Molecular simulation and mechanism of viscosity reduction and upgrading of super heavy oil. Pet. J. 2015, 36, 355-360.

32. Li, Q.; Pu, W.F.; Wang, Y.B.; Geng, X.F. Synthesis and Properties of AM/AMPS Copolymers. Appl. Chem. Ind. 2012, 41, $300-303$.

33. Zhang, S.P. Synthesis of Amphiphilic Polymers Containing Quaternary Quaternary Ammonium Salts and Their Application in Viscosity Reduction of Heavy Oils; Shandong University: Jinan, China, 2017.

34. Li, Y.Y.; Wu, Q.; Xu, P.; Ma, A.Q.; Hou, Y.F. Preparation and performance optimization of a new viscosity-reducing agent for crude oil based on molecular simulation. Chem. Ind. Eng. Progress 2020, 12, 5234-5242.

35. Zhu, J.; Li, C.X.; Xin, P.G. The effect of structure of viscosity reducer to viscosity reducing effect for heavy oil. J. Petrochem. Univ. 2011, 24, 39-42.

36. Cui, Q.; Zhang, C.Q.; Xiu, J.X.; Xiu, S.M.; Lu, L.L. Molecular dynamics simulation of viscosity reduction mechanism of heavy oil asphaltene colloid. J. Shandong Univ. (Eng. Sci.) 2017, 47, 123-130.

37. Khabaz, F.; Khare, R. Effect of chain architecture on the size, shape, and intrinsic viscosity of chains in polymer solutions: A molecular simulation study. J. Chem. Phys. 2014, 141, 214904. [CrossRef] [PubMed] 